\title{
Région touchée par la catastrophe dans le nord de Sumatra: le manque de médecins est comblé
}

Dans le $n^{\circ} 3$ du Bulletin des médecins suisses de cette année, M. O. Seeger, de la fondation PanEco, a publié un article évoquant un grave manque de médecins dans la région sinistrée du nord de Sumatra. Il y est question d'un médecin d'une organisation indonésienne non gouvernementale nommée YEL, qui déclare urgent d'envoyer dans la région touchée par la catastrophe au moins 500 médecins et 700 personnes soignantes.

Les trois soussignés, qui étaient personnellement dans cette région entre le 3 et le 10 janvier derniers, dont trois jours à Sumatra, ne sauraient soutenir cet appel. En effet, les rapports les plus actuels sur la situation, reçus par l'intermédiaire de leurs canaux d'information, ainsi que les contacts directs avec des médecins sur place, y compris ceux de PanEco et autres ONG, donnent à penser que s'il existe un besoin ponctuel en médecins et en soignants, c'est surtout pour relayer les équipes qui s'en vont. Ces passages de témoin se passent normalement sans problèmes au sein même des organisations.

Le nombre de personnes ayant besoin de soins urgents après le raz-de-marée a en toute logique beaucoup reculé. Il s'agit désormais surtout d'assurer l'assistance médicale de base et les soins hospitaliers des survivants sur place, d'aider les sans-abris et les personnes qui ont dû fuir et de reconstruire les structures de la santé publique. Dans une deuxième phase, une autre exigence consistera dans la réadaptation des nombreuses victimes qui ne s'en sont sorties que grâce à une amputation .

L'article précité a suscité une vague de demandes aux soussignés de la part de médecins et d'équipes soignantes prêtes à apporter leur aide, qui ont tous dû être informés de l'inutilité de leur démarche pour le moment, souvent à leur déception.

Actuellement, la situation est sous constante observation de l'OMS, mandatée par l'ONU pour assurer la coordination internationale en matière de santé, ce qui revient à dire que les besoins exacts sont communiqués et transmis à qui de droit par l'aide humanitaire.

Toni Frisch, délégué à l'aide humanitaire

Daniel Biedermann, directeur de la Croix-Rouge suisse

Gianpiero A. Lupi, chef des troupes sanitaires, médecin en chef de l'armée 